\title{
Bernoulli Wavelets Method for Solution of Fractional Differential Equations in a Large Interval
}

\author{
Keshavarz E., "Ordokhani Y.; \\ Faculty of Mathematical Sciences, Alzahra University \\ Received: 18 Nov 2013 \\ Revised: 10 Nov 2014
}

\begin{abstract}
In this paper, Bernoulli wavelets are presented for solving (approximately) fractional differential equations in a large interval. Bernoulli wavelets operational matrix of fractional order integration is derived and utilized to reduce the fractional differential equations to system of algebraic equations. Numerical examples are carried out for various types of problems, including fractional Van der Pol and Bagley-Torvik equations for the application of the method. Illustrative examples are presented to demonstrate the efficiency and accuracy of the proposed method.
\end{abstract}

Keywords: Bernoulli wavelet, Fractional calculus, Differential equations, Block pulse function, Van der Pol equation, Bagley-Torvik equation, Caputo derivative, Operational matrix, Numerical solution

\section{Introduction}

In recent decades, applied scientists and engineers have realized that fractional differential equations (FDEs) provide a better approach to describing the complex phenomena in nature such as non-Brownian motion, signal processing, systems identification, control, viscoelastic materials and polymers [1-3].

In general, it is not easy to derive the analytical solutions to most of the FDEs. Therefore, it is vital to develop some reliable and efficient techniques to solve FDEs. Numerical solution of FDEs has attracted considerable attention from many researchers. During the past decades, an increasing number of numerical schemes have been developed. These methods include fractional linear multi-step methods [4,5], the Adomian decomposition method [6-8], variational iteration method [9, 10], operational matrix method [11-22] and Homotopy analysis method [23-26].

Numerical solutions of the FDEs in a large interval have been investigated by some researchers. There are few references on the solution for fractional Van der Pol

\footnotetext{
"Corresponding author:

ordokhani@alzahra.ac.ir
} 
equations [27, 28] and Bagley-Torvik equations [29]. In this paper we solve the two above noted equations. We introduce a new direct computational method to solve them. The method consists of using Bernoulli wavelet operational matrix of fractional order integration to reduce the FDEs to a set of algebraic equations. For approximating an arbitrary time functions the advantages of Bernoulli polynomials $\beta_{m}(t), m=0,1,2, \ldots$ where $0 \leq t \leq 1$ over shifted Legendre polynomials are given in [30-32].

The rest of the paper is organized as follows: in section 2, basic definitions of fractional calculus, Bernoulli wavelets and their properties are introduced. In section 3, how to derive Bernoulli wavelet operational matrix of fractional order integration is given. Illustrative examples are given in section 4 to demonstrate the application of operational matrix of fractional order integration for Bernoulli wavelets. Finally, we present the conclusion in section 5 .

\section{Preliminaries and notations}

\section{The fractional integral and derivative}

In this section, we first state some definitions and basic properties regarding fractional derivatives and integral. There are various definitions of fractional integration and differentiation, such as Grunwald-Letnikov's definition and Riemann-Liouville's definition. To solve differential equations (both classical and fractional), we need to specify additional conditions in order to produce a unique solution. For the case of the Caputo FDEs, the additional conditions are just the traditional conditions, which are akin to those of classical differential equations, and are therefore familiar to us. In contrast, for the Riemann-Liouville FDEs, the additional conditions constitute certain fractional derivatives (and/or integrals) of the unknown solution at the initial point $t=0$ , which are functions of $t$. These initial conditions are not physical, furthermore, it is not clear how such quantities are to be measured from experiment, say, so that they can be appropriately assigned in an analysis. For more details see [33]. Therefore, we use the fractional derivatives in the Caputo sense.

Definition 1. We define

$$
\begin{gathered}
C_{\mu}=\left\{f(t) \mid f(t)>0, t>0, f(t)=t^{p} f_{1}(t)\right\}, \text { where } p>\mu \text { and } f_{1}(t) \in C[0, \infty), \\
C_{\mu}{ }^{n}=\left\{f(t) \mid f^{(n)}(t) \in C_{\mu}\right\}, \text { where } n \in \mathbb{N} \text { and } \mu \in \mathbb{R} .
\end{gathered}
$$

Definition 2. The Riemann-Liouville fractional integral operator of order $q \geq 0$, of a function $f(t) \in C_{\mu}, \mu \geq-1$, is defined as [34] 


$$
I^{q} f(t)= \begin{cases}\frac{1}{\Gamma(q)} \int_{0}^{t} \frac{f(s)}{(t-s)^{1-q}} d s, & q>0, \\ f(t), & q=0 .\end{cases}
$$

For the Riemann-Liouville fractional integral we have

$$
I^{q} t^{v}=\frac{\Gamma(v+1)}{\Gamma(v+1+q)} t^{v+q}, \quad v>-1 .
$$

The Riemann-Liouville fractional integral is a linear operation, namely:

$$
I^{q}(\lambda f(t)+\mu g(t))=\lambda I^{q} f(t)+\mu I^{q} g(t),
$$

where $\lambda$ and $\mu$ are constants.

Definition 3. Caputo's fractional derivative of order $q$, for the function $f(t) \in C_{-1}{ }^{n}$, is defined as [35]

$$
D^{q} f(t)=\frac{1}{\Gamma(n-q)} \int_{0}^{t} \frac{f^{(n)}(s)}{(t-s)^{q+1-n}} d s, \quad n-1<q \leq n, n \in \mathbb{N},
$$

where $q>0$ is the order of the derivative and $n$ is the smallest integer greater than or equal $q$.

For the Caputo derivative we have the following two basic properties [36]

$$
D^{q} I^{q} f(t)=f(t)
$$

and

$$
I^{q} D^{q} f(t)=f(t)-\sum_{i=0}^{n-1} f^{(i)}(0) \frac{t^{i}}{i !}
$$

\section{The properties of Bernoulli wavelets}

\subsection{Wavelets and Bernoulli wavelets}

Wavelets are a family of functions constructed from dilation and translation of a single function called the mother wavelet. When the dilation parameter $a$ and the translation parameter $b$ vary continuously we have the following family of continuous wavelets as [37]

$$
\psi_{a, b}(t)=|a|^{\frac{-1}{2}} \psi\left(\frac{t-b}{a}\right), \quad a, b \in \mathbb{R}, a \neq 0 .
$$

If we restrict the parameters $a$ and $b$ to discrete values as $a=a_{0}^{-k}, b=n b_{0} a_{0}^{-k}, a_{0}>1$, $b_{0}>1$, where $n$ and $k$ are positive integers, the family of discrete wavelets are defined as

$$
\psi_{k, n}(t)=\left|a_{0}\right|^{\frac{k}{2}} \psi\left(a_{0}^{k} t-n b_{0}\right)
$$


where $\psi_{k, n}(t)$ form a wavelet basis for $L^{2}(\mathbb{R})$.

Bernoulli wavelets $\psi_{n, m}(t)=\psi(k, \hat{n}, m, t)$ have four arguments, $\hat{n}=n-1, n=1,2,3, \ldots, 2^{k-1}$ $k$ can assume any positive integer, $m$ is the order for Bernoulli polynomials and $t$ is the normalized time. We define them on the interval $[0,1)$ as follows [38]

$$
\psi_{n, m}(t)=\left\{\begin{array}{cc}
2^{\frac{k-1}{2}} \tilde{\beta}_{m}\left(2^{k-1} t-\hat{n}\right), & \frac{\hat{n}}{2^{k-1}} \leq t<\frac{\hat{n}+1}{2^{k-1}}, \\
0, & \text { otherwise }
\end{array}\right.
$$

with

$$
\tilde{\beta}_{m}(t)=\left\{\begin{array}{cc}
1, & m=0, \\
\frac{1}{\sqrt{\frac{(-1)^{m-1}(m !)^{2}}{(2 m) !}} \alpha_{2 m}} \beta_{m}(t), & m>0,
\end{array}\right.
$$

where $m=0,1,2, \ldots, M-1, n=1,2, \ldots, 2^{k-1}$. The coefficient $\frac{1}{\sqrt{\frac{(-1)^{m-1}(m !)^{2}}{(2 m) !} \alpha_{2 m}}}$ is for

normality, the dilation parameter is $a=2^{-(k-1)}$ and the translation parameter $b=\hat{n} 2^{-(k-1)}$. Here, $\beta_{m}(t)$ are the well-known Bernoulli polynomials of order $m$ which can be defined by [39]

$$
\beta_{m}(t)=\sum_{i=0}^{m}\left(\begin{array}{c}
m \\
i
\end{array}\right) \alpha_{m-i} t^{i}
$$

where $\alpha_{i}, i=0,1, \ldots, m$ are Bernoulli numbers. These numbers are a sequence of signed rational numbers which arise in the series expansion of trigonometric functions [40] and can be defined by the identity

$$
\frac{t}{e^{t}-1}=\sum_{i=0}^{\infty} \alpha_{i} \frac{t^{i}}{i !}
$$

The first few Bernoulli numbers are

$$
\alpha_{0}=1, \alpha_{1}=\frac{-1}{2}, \alpha_{2}=\frac{1}{6}, \alpha_{4}=\frac{-1}{30}, \ldots,
$$

with $\alpha_{2 i+1}=0, \quad i=1,2,3, \ldots$

The first few Bernoulli polynomials are

$$
\beta_{0}(t)=1, \quad \beta_{1}(t)=t-\frac{1}{2}, \quad \beta_{2}(t)=t^{2}-t+\frac{1}{6}, \quad \beta_{3}(t)=t^{3}-\frac{3}{2} t^{2}+\frac{1}{2} t, \ldots
$$

These polynomials satisfy the following formula [40]

$$
\int_{0}^{1} \beta_{n}(t) \beta_{m}(t) d t=(-1)^{n-1} \frac{m ! n !}{(m+n) !} \alpha_{n+m}, \quad m, n \geq 1 .
$$


According to [41], Bernoulli polynomials form a complete basis over the interval [0,1].

\subsection{Function approximation}

Suppose that $\left\{\psi_{10}(t), \psi_{11}(t), \ldots, \psi_{2^{k-1} M_{-1}}(t)\right\} \subset L^{2}[0,1]$ is the set of Bernoulli wavelets,

$$
Y=\operatorname{span}\left\{\psi_{10}(t), \psi_{11}(t), \ldots, \psi_{1 M-1}(t), \psi_{20}(t), \ldots, \psi_{2 M-1}(t), \ldots, \psi_{2^{k-1} 0}(t), \ldots, \psi_{2^{k-1} M-1}(t)\right\},
$$

and $f(t)$ be an arbitrary element in $L^{2}[0,1]$. Since $Y$ is a finite dimensional vector space, $f(t)$ has the best approximation out of $Y$ such as $f_{0}(t) \in Y$, that is

$$
\forall y(t) \in Y,\left\|f(t)-f_{0}(t)\right\| \leq\|f(t)-y(t)\| .
$$

Since $f_{0}(t) \in Y$, there exists unique coefficients $c_{10}, c_{11}, \ldots, c_{2^{k-1} M-1}$ such that

$$
f(t) \simeq f_{0}(t)=\sum_{n=1}^{2^{k-1}} \sum_{m=0}^{M-1} c_{n m} \psi n m(t)=C^{T} \Psi(t),
$$

where $T$ indicates transposition, $C$ and $\Psi(t)$ are $2^{k-1} M \times 1$ matrices given by

$$
\begin{gathered}
C=\left[c_{10}, c_{11}, \ldots, c_{1 M-1}, c_{20}, \ldots, c_{2 M-1}, \ldots, c_{2^{k-1} 0}, \ldots, c_{2^{k-1} M-1}\right]^{T}, \\
\Psi(t)=\left[\psi_{10}(t), \psi_{11}(t), \ldots, \psi_{1 M-1}(t), \psi_{20}(t), \ldots, \psi_{2 M-1}(t), \ldots, \psi_{2^{k-1} 0}(t), \ldots, \psi_{2^{k-1} M-1}(t)\right]^{T} .
\end{gathered}
$$

Using Eq. (3) we obtain

$$
f_{i j}=<\sum_{n=1}^{2^{k-1}} \sum_{m=0}^{M-1} c_{n m} \psi_{n m}(t), \psi_{i j}(t)>=\sum_{n=1}^{2^{k-1}} \sum_{m=0}^{M-1} c_{n m} d_{n m}^{i j}, i=1,2, \ldots, 2^{k-1}, j=0,1, \ldots, M-1,
$$

that $\left.f_{i j}=<f(t), \psi_{i j}(t)\right\rangle, \quad d_{n m}^{i j}=\left\langle\psi_{n m}(t), \psi_{i j}(t)\right\rangle$, and $<,>$ denotes inner product.

Therefore,

$$
\begin{gathered}
f_{i j}=C^{T}\left[d_{10}^{i j}, d_{11}^{i j}, \ldots, d_{1 M-1}^{i j}, d_{20}^{i j}, \ldots, d_{2 M-1}^{i j}, \ldots, d_{2^{k-1} 0}^{i j}, \ldots, d_{2^{k-1} M-1}^{i j}\right]^{T}, \\
i=1,2, \ldots, 2^{k-1}, j=0,1, \ldots, M-1,
\end{gathered}
$$

or

$$
F^{T}=C^{T} D
$$

where

$$
F=\left[f_{10}, f_{11}, \ldots, f_{1 M-1}, f_{20}, \ldots, f_{2 M-1}, \ldots, f_{2^{k-1} 0}, \ldots, f_{2^{k-1} M-1}\right]^{T},
$$

and

$$
D=\left[d_{n m}^{i j}\right],
$$

where $D$ is a matrix of order $2^{k-1} M \times 2^{k-1} M$ and is given by

$$
D=\int_{0}^{1} \Psi(t) \Psi^{T}(t) d t
$$

The matrix $D$ in Eq. (4) can be calculated by using Eq. (2) in each interval $n=1, \ldots, 2^{k-1}$. For example with $k=2$ and $M=3, D$ is the identity matrix and for 
$k=2$ and $M=4$ we have

$$
D=\left[\begin{array}{cccccccc}
1 & 0 & 0 & 0 & 0 & 0 & 0 & 0 \\
0 & 1 & 0 & -\sqrt{\frac{7}{10}} & 0 & 0 & 0 & 0 \\
0 & 0 & 1 & 0 & 0 & 0 & 0 & 0 \\
0 & -\sqrt{\frac{7}{10}} & 0 & 1 & 0 & 0 & 0 & 0 \\
0 & 0 & 0 & 0 & 1 & 0 & 0 & 0 \\
0 & 0 & 0 & 0 & 0 & 1 & 0 & -\sqrt{\frac{7}{10}} \\
0 & 0 & 0 & 0 & 0 & 0 & 1 & 0 \\
0 & 0 & 0 & 0 & 0 & -\sqrt{\frac{7}{10}} & 0 & 1
\end{array}\right] .
$$

Therefore, $C^{T}$ in Eq. (3) is given by

$$
C^{T}=F^{T} D^{-1}
$$

\section{Bernoulli wavelet operational matrix of the fractional integration}

In this section, we define the Bernoulli wavelet matrix. Then, by using the BlockPulse operational matrix of fractional integration, we derive the Bernoulli wavelet operational matrix of the fractional integration.

Taking the collocation points as following:

$$
t_{i}=\frac{2 i-1}{2^{k} M}, \quad i=1,2, \ldots, 2^{k-1} M,
$$

we define the Bernoulli wavelet matrix $\Phi_{m^{\prime} \times m^{\prime}}$ as:

$$
\Phi \triangleq\left[\Psi\left(\frac{1}{2 m^{\prime}}\right), \Psi\left(\frac{3}{2 m^{\prime}}\right), \ldots, \Psi\left(\frac{2 m^{\prime}-1}{2 m^{\prime}}\right)\right],
$$

where $m^{\prime}=2^{k-1} M$. For example, when $k=2$ and $M=3$ the Bernoulli wavelet matrix is expressed as

$$
\Phi=\left[\begin{array}{cccccc}
1.41421 & 1.41421 & 1.41421 & 0 & 0 & 0 \\
-1.63299 & 0 & 1.63299 & 0 & 0 & 0 \\
0.527046 & -1.58114 & 0.527046 & 0 & 0 & 0 \\
0 & 0 & 0 & 1.41421 & 1.41421 & 1.41421 \\
0 & 0 & 0 & -1.63299 & 0 & 1.63299 \\
0 & 0 & 0 & 0.527046 & -1.58114 & 0.527046
\end{array}\right] .
$$

Now, we define an $m^{\prime}$-set of Block-Pulse functions (BPFs) as 


$$
b_{i}(t)=\left\{\begin{array}{l}
1, \quad \frac{i}{m^{\prime}} \leq t<\frac{i+1}{m^{\prime}}, \\
0, \quad \text { otherwise, }
\end{array}\right.
$$

where $i=0,1,2, \ldots, m^{\prime}-1$. BPFs have the following useful property

$$
b_{i}(t) b_{j}(t)= \begin{cases}0, & i \neq j, \\ b_{i}(t), & i=j .\end{cases}
$$

Bernoulli wavelets may be expanded into an $m^{\prime}$-term BPFs as

$$
\Psi(t)=\Phi B(t),
$$

where $B(t) \triangleq\left[b_{0}(t), b_{1}(t), \ldots, b_{m^{\prime}-1}(t)\right]^{T}$. Kilicman and Al Zhour have given the BlockPulse operational matrix of the fractional integration $F^{(q)}$ as following [42]:

$$
I^{q} B(t)=F^{(q)} B(t),
$$

where

$$
F^{(q)}=\frac{1}{m^{\prime q}} \frac{1}{\Gamma(q+2)}\left[\begin{array}{ccccc}
1 & \xi_{1} & \xi_{2} & \cdots & \xi_{m^{\prime}-1} \\
0 & 1 & \xi_{1} & \cdots & \xi_{m^{\prime}-2} \\
0 & 0 & 1 & \cdots & \xi_{m^{\prime}-3} \\
0 & 0 & 0 & \ddots & \vdots \\
0 & 0 & 0 & 0 & 1
\end{array}\right],
$$

with $\xi_{k}=(k+1)^{q+1}-2 k^{q+1}+(k-1)^{q+1}$.

We now derive the Bernoulli wavelet operational matrix of fractional integration. Let

$$
I^{q} \Psi(t)=P^{(q)} \Psi(t),
$$

where the matrix $P^{(q)}$ is called the Bernoulli wavelet operational matrix of fractional integration. Using Eqs. (6) and (7) we get

$$
I^{q} \Psi(t)=I^{q} \Phi B(t)=\Phi I^{q} B(t)=\Phi F^{(q)} B(t) .
$$

From Eqs. (8) and (9) we have

$$
P^{(q)} \Psi(t)=P^{(q)} \Phi B(t)=\Phi F^{(q)} B(t) .
$$

Then, the Bernoulli wavelet operational matrix of fractional integration $P^{(q)}$ is given by

$$
P^{(q)}=\Phi F^{(q)} \Phi^{-1} \text {. }
$$

In particular, for $k=2, M=3, q=0.5$, the Bernoulli wavelet operational matrix of fractional integration $P^{(q)}$ is expressed as

$$
P^{(0.5)}=\left[\begin{array}{cccccc}
0.528223 & 0.181881 & -0.0297821 & 0.443844 & -0.087099 & 0.0256378 \\
-0.14516 & 0.224295 & 0.132924 & 0.0798823 & -0.0449052 & 0.0198105 \\
-0.0598166 & -0.096441 & 0.168799 & -0.0417244 & -0.000185889 & 0.00286805 \\
0 & 0 & 0 & 0.528223 & 0.181881 & -0.0297821 \\
0 & 0 & 0 & -0.14516 & 0.224295 & 0.132924 \\
0 & 0 & 0 & -0.0598166 & -0.096441 & 0.168799
\end{array}\right] .
$$

It should be noted that the operational matrix $P^{(q)}$ contains many zero entries. This 
phenomena makes calculations fast. The calculation for the matrix $P^{(q)}$ is carried out for fixed $k, M$, and is used to solve fractional order as well as integer order differential equations.

\section{Illustrative examples}

To demonstrate the effectiveness of the method, we consider some fractional differential equations. We use Mathematica ver. 7.0 software to solve following examples.

Example 1. Consider the damped Van der Pol equation with fractional damping, which is governed by the studies of Juhn-Horng Chen and Wei-Ching Chen [43] as following

$$
\begin{aligned}
\ddot{x}(t)+\mu\left(x^{2}(t)-1\right) D^{q} x(t)+x(t) & =\operatorname{asin}(\omega t), 0<q<2, \\
x(0) & =0, \\
\dot{x}(0) & =0,
\end{aligned}
$$

the second initial condition is for $q>1$ only. The initial conditions are homogeneous, $\mu$ is the damping parameter, $a$ the amplitude of periodic forcing, $\omega$ denotes the forcing frequency and $D^{q}$ the differential operator that denotes the $q^{\text {th }}$ derivative of the related function with respect to $t$.

Let

$$
D^{2} x(t) \approx C^{T} \Psi(t),
$$

by using Eqs. (1), (6), (8), (10) and (12) we have

$$
D^{q} x(t)=\left(I^{2-q} D^{2} x\right)(t)=C^{T} P^{(2-q)} \Psi(t)=C^{T} \Phi F^{(2-q)} \Phi^{-1} \Psi(t),
$$

and

$$
x(t)=C^{T} P^{(2)} \Psi(t)+x(0)+\dot{x}(0) t=C^{T} P^{(2)} \Phi B(t)=A_{1} B(t),
$$

where

$$
A_{1}=C^{T} P^{(2)} \Phi=\left[a_{0}, a_{1}, \ldots, a_{m^{\prime}-1}\right] .
$$

By using Eqs. (5) and (15), we have

$$
\begin{gathered}
{[x(t)]^{2}=\left[A_{1} B(t)\right]^{2}=\left[a_{0} b_{0}(t)+a_{1} b_{1}(t)+\ldots+a_{m^{\prime}-1} b_{m^{\prime}-1}(t)\right]^{2}} \\
=a_{0}^{2} b_{0}(t)+a_{1}^{2} b_{1}(t)+\ldots+a_{m^{\prime}-1}^{2} b_{m^{\prime}-1}(t)=A_{2} B(t),
\end{gathered}
$$

where

$$
A_{2}=\left[a_{0}^{2}, a_{1}^{2}, \ldots, a_{m^{\prime}-1}^{2}\right] .
$$

Similarly, the input signal $\operatorname{asin}(\omega t)$ may be expanded by the Bernoulli wavelets as

$$
\operatorname{asin}(\omega t)=F^{T} \Psi(t),
$$

where $F^{T}$ is a known constant vector. Substituting Eqs. (13)-(16) in Eq. (11), we have 


$$
\begin{aligned}
& C^{T} \Psi(t)+\mu A_{2} B(t) C^{T} \Phi F^{(2-q)} \Phi^{-1} \Psi(t)-\mu C^{T} \Phi F^{(2-q)} \Phi^{-1} \Psi(t) \\
& \quad+C^{T} P^{(2)} \Psi(t)-F^{T} \Psi(t)=0 .
\end{aligned}
$$

To find the solution $x(t)$, we first collocate Eq. (17) at the points $t_{i}=\frac{2 i-1}{2^{k} M}$, $i=1,2, \ldots, 2^{k-1} M$. These equations generate $2^{k-1} M$ algebraic equations which can be solved by using Newton's iterative method. Consequently, $x(t)$ given in Eq. (15) can be calculated approximately.

We assume that the parametric values are $\mu=1.02, a=1.31, \omega=0.5$, and solve the fractional damped Van der Pol for $q=0.5$. It is worth noticing that the method introduced above can only solve Eq. (11) for $t \in[0,1)$. That is because Bernoulli wavelets are defined on interval $[0,1)$. However, the variable $t$ of Eq. (11) is defined on interval $[0,4)$, we should turn $\Psi(t)$ into $\Psi\left(\frac{t}{4}\right)$ in the discrete procedure. Figures 1 and 2 show the approximate solution obtained by our method with $k=3, M=4$ within interval $[0,1)$ and $[0,4)$, respectively. The numerical solution is in perfect agreement with the solutions of [27] and [28].

Example 2. Consider the Bagley-Torvik equation that governs the motion of a rigid plate immersed in a Newtonian fluid

$$
\begin{aligned}
a \ddot{x}(t)+b D^{1.5} x(t)+c x(t) & =f(t), \quad t>0, \\
x(0) & =\dot{x}(0)=0,
\end{aligned}
$$

where

$$
f(t)=8[u(t)-u(t-1)],
$$

and $u(t)$ is the Heaviside Step function.

The analytical solution of Eq. (18) obtained by Pondlubny is $x(t)=8\left[x_{u}(t)-x_{u}(t-1)\right]$, [2].

where

$$
x_{u}(t)=u(t)\left[\frac{1}{a} \sum_{i=0}^{\infty} \frac{(-1)^{i}}{i !}\left(\frac{c}{a}\right)^{i} t^{2(i+1)} E_{\frac{1}{2}, \frac{3}{2} i+3}^{(i)}\left(\frac{-b}{a} t^{\frac{1}{2}}\right)\right],
$$

and $E_{\xi, \gamma}(t)$ is the Mittag-Leffler function in two parameters $\xi, \gamma$ :

$$
E_{\xi, \gamma}^{(i)}(t)=\frac{d^{i}}{d t^{i}} E_{\xi, \gamma}(t)=\sum_{j=0}^{\infty} \frac{(i+j) ! t^{j}}{j ! \Gamma(\xi j+\xi i+\gamma)}, \quad i=0,1,2, \ldots
$$

Integrating Eq. (18), we have 


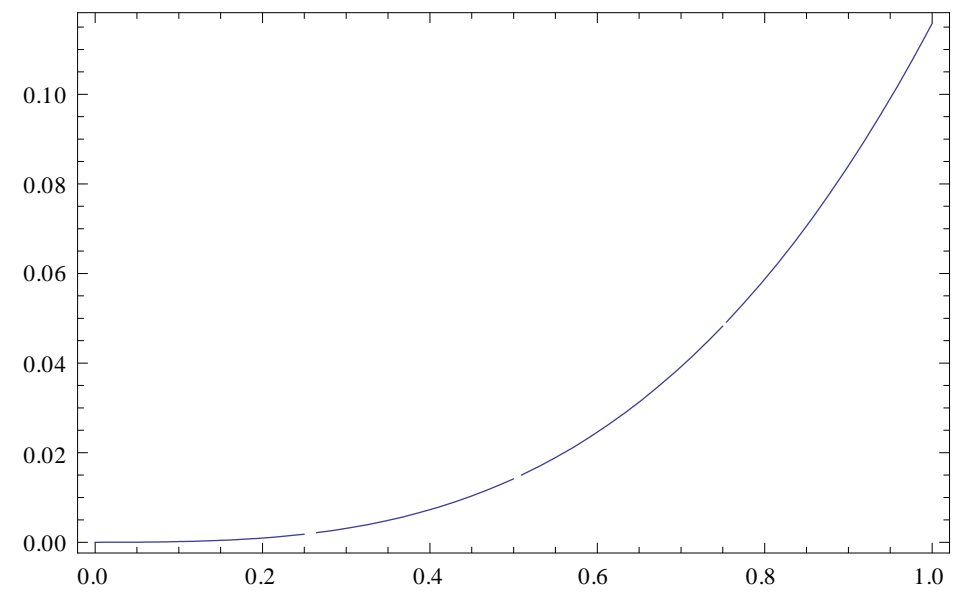

Figure 1. Approximate solution within interval $[0,1)$, for Example 4.1

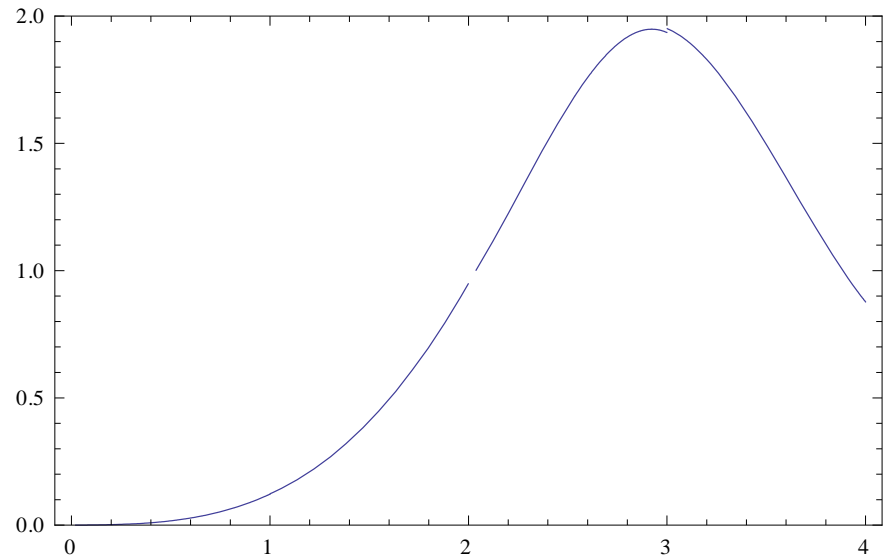

Figure 2. Approximate solution within interval $[0,4)$, for Example 4.1

$$
a[x(t)-x(0)-t \dot{x}(0)]+b I^{0.5} x(t)+c I^{2} x(t)=I^{2} f(t) .
$$

Let

$$
x(t) \approx C^{T} \Psi(t) .
$$

Similarly, the input signal $f(t)$ may be expanded by the Bernoulli wavelet as

$$
f(t) \approx F^{T} \Psi(t) \text {. }
$$

Substituting Eqs. (19), (21) and (22) into (20), we have

$$
a C^{T} \Psi(t)+b C^{T} I^{0.5} \Psi(t)+c C^{T} I^{2} \Psi(t)-F^{T} I^{2} \Psi(t)=0,
$$

then by using Eq. (8) we have

$$
a C^{T} \Psi(t)+b C^{T} P^{(0.5)} \Psi(t)+c C^{T} P^{(2)} \Psi(t)-F^{T} P^{(2)} \Psi(t)=0,
$$

or

$$
a C^{T}+b C^{T} P^{(0.5)}+c C^{T} P^{(2)}-F^{T} P^{(2)}=0,
$$

then

$$
C^{T}=F^{T} P^{(2)}\left[a I+b P^{(0.5)}+c P^{(2)}\right]^{-1} .
$$


Finding the unknown coefficient vector $C, x(t)$ given in Eq. (21) can be calculated.

We assume that $a=1, b=0.5, c=0.5$, and solve Eq. (18) with $k=4, M=4$. Figure 3 shows the numerical solution (on the interval $[0,20)$ method is the same as Example 4.1) that is in very good agreement with the exact solution. This demonstrates the importance of our numerical scheme in solving nonlinear multi-order fractional differential equations. Also, Table 1 shows the absolute error of $x(t)$ for our method and Haar wavelet [29].

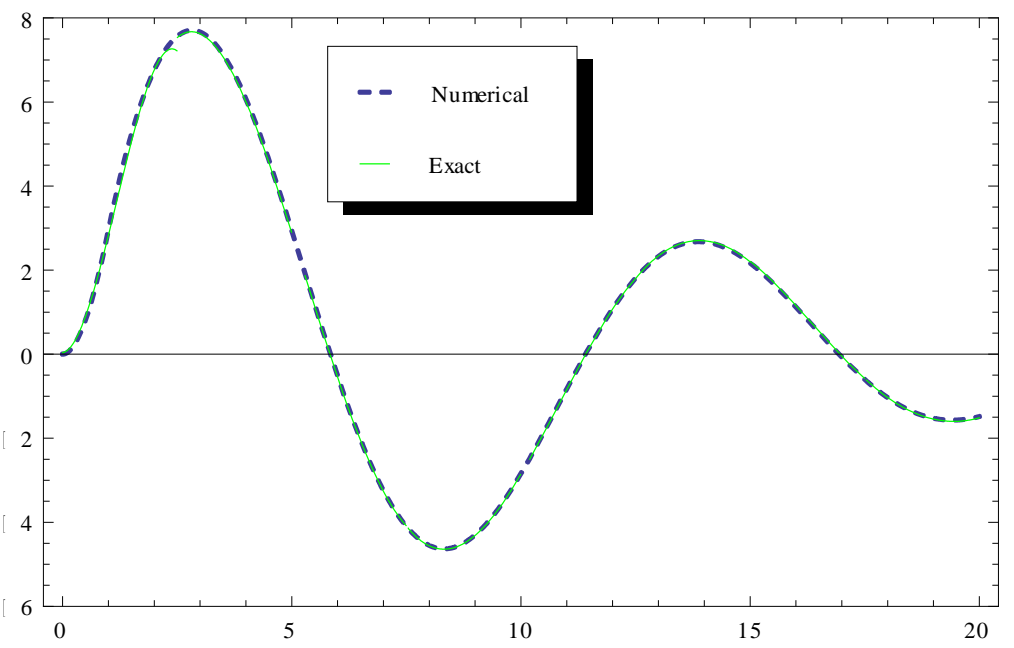

Figure 3. Numerical and exact solution within interval $[0,20)$, for Example 4.2

Table 1. Absolute error of $x(t)$, for Example 4.2

\begin{tabular}{|c|c|c|}
\hline $\mathrm{t}$ & Haar wavelet [29] & Bernoulli wavelet \\
\hline 1 & 0.585974 & 0.124625 \\
\hline 2 & 0.77707 & 0.00429792 \\
\hline 3 & 0.61926 & 0.0414732 \\
\hline 4 & 0.184014 & 0.0645971 \\
\hline 5 & 0.41339 & 0.0347576 \\
\hline 6 & 0.966783 & 0.029914 \\
\hline 7 & 1.26268 & 0.0161954 \\
\hline 8 & 1.17045 & 0.00931825 \\
\hline 9 & 0.697989 & 0.00940264 \\
\hline
\end{tabular}

\section{Example 3. Consider the equation}

$$
D^{2.2} x(t)+1.3 D^{1.5} x(t)+2.6 x(t)=\sin (2 t)
$$

with the initial conditions

$$
x(0)=\dot{x}(0)=\ddot{x}(0)=0 .
$$

This problem was solved in [44]. Figure 4 shows the behavior of the numerical solution on the interval $[0,10)$ with $k=5, M=4$. Our result is in good agreement with the numerical result obtained by [43]. 


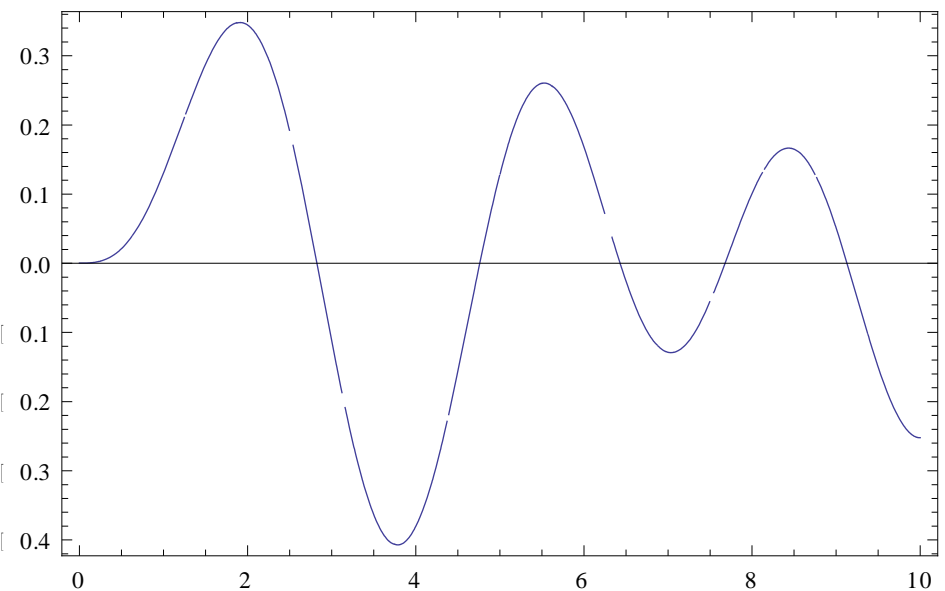

Figure 4. Approximate solution within interval $[0,10)$, for Example 4.3

\section{Conclusion}

A general formulation for the Bernoulli wavelet operational matrix of fractional integration has derived. This matrix is used to approximate numerical solution of FDEs. The advantage of this method is that it transforms the problem into algebraic matrix equation so that the computation is simple and it is computer oriented method. It shows simplicity and effectiveness of this method. The solution obtained using the suggested method confirems that this approach can solve the problems effectively.

\section{References}

1. Das S., "Functional Fractional Calculus for System Identification and Controls", New York: Springer (2008).

2. Kilbas A. A., Srivastava H. M., Trujillo J. J., "Theory and Applications of Fractional Differential Equations", San Diego: Elsevier (2006).

3. Podlubny I., "Fractional Differential Equations", in: Mathematics in Science and Engineering, vol. 198. San Diego, Calif: Academic Press Inc. (1999).

4. Ford N. J., Connolly A. J., "Systems-based decomposition schemes for the approximate solution of multi-term fractional differential equations", Journal of Computational and Applied Mathematics, 229 (2009) 382-391.

5. Ford N. J. , Simpson A. C., "The numerical solution of fractional differential equations: speed versus accuracy", Numerical Algorithms, 26 (2001) 333-346.

6. Duan J. S., Chaolu T., Rach R., Lu L., "The Adomian decomposition method with convergence acceleration techniques for nonlinear fractional differential equations", 
Computers and Mathematics with Applications, 66(5) (2013) 728-736.

7. Duan J., Chaolu T. , Rach R., "Solutions of the initial value problem for nonlinear fractional ordinary differential equations by the Rach-Adomian-Meyers modified decomposition method", Applied Mathematics and Computation, 218(17) (2012) 8370-8392.

8. Song L., Wang W., "A new improved Adomian decomposition method and its application to fractional differential equations", Applied Mathematical Modelling, 37 (3) (2013) 15901598.

9. Wu G. C., "A fractional variational iteration method for solving fractional nonlinear differential equations", Computers and Mathematics with Applications, 61(8) (2011) 21862190.

10. Yang S., Xiao A., Su H., "Convergence of the variational iteration method for solving multiorder fractional differential equations", Computers and Mathematics with Applications, 60(10) (2010) 2871-2879.

11. Saadatmandi A., Dehghan M., "A new operational matrix for solving fractional-order differential equations", Computers and Mathematics with Applications, 59 (2010) 13261336.

12. Hwang C., Shih Y. P., "Laguerre operational matrices for fractional calculus and applications", International Journal of Control, 34(3) (1981) 577-584.

13. Wang C. H., "On the generalization of block-pulse operational matrices for fractional and operational calculus", Journal of the Franklin Institute, 315(2) (1983) 91-102.

14. Lakestani M., Dehghan M., Irandoust-pakchin S., "The construction of operational matrix of fractional derivatives using B-spline functions", Communications in Nonlinear Science and Numerical Simulation, 17 (2012) 1065-1064.

15. Wang M. L., Chang R. Y., Yang S. Y., "Generalization of generalized orthogonal polynomial operational matrices for fractional and operational calculus", International Journal of Systems Science, 18(5) (1987) 931-943.

16. Khader M. M., "Numerical solution of nonlinear multi-order fractioanl defferential equations by implementation of the operatioanl matrix of fractional derivative", Studies in Nonlinear Sciences, 2 (1) (2011) 5-12.

17. Rehman M., Ali Khan R., "A numerical method for solving boundary value problems for fractional differential equations", Applied Mathematical Modelling, 36 (2012) 894-907. 
18. Chang R. Y., Chen K. C., Wang M., "Modified Laguerre operational matrices for fractional calculus and applications", International Journal of Systems Science, 16(9) (1985) 1163 1172 .

19. Yuanlu L., Ning S., "Numerical solution of fractional differential equations using the generalized block-pulse operational matrix", Computers and Mathematics with Applications, 216 (2010) 1046-1054.

20. Atanackovic T. M., Stankovic B., "On a numerical scheme for solving differential equations of fractional order", Mechanics Research Communications, 35 (7) (2008) 429-438.

21. Yuanlu L., Ning S., "Numerical solution of fractional differential equations using the generalized block-pulse operational matrix", Computers and Mathematics with Applications, 216 (2010) 1046-1054.

22. Yuanlu L., Weiwei Z., "Haar wavelet operational matrix of fractional order integration and its applications in solving the fractioanl order differntial equations", Applied Mathematics and Computation, 216 (8) (2010) 2276-2285.

23. Odibat Z., Momani S., Xu H., "A reliable algorithm of homotopy analysis method for solving nonlinear fractional differential equations", Applied Mathematical Modelling, 34 (3) (2010) 593-600.

24. Hosseinnia S. H., Ranjbar A., Momani S., "Using an enhanced homotopy perturbation method in fractional differential equations via deforming the linear part", Computers and Mathematics with Applications, 56 (12) (2008) 3138-3149.

25. Jafari H., Das S., Tajadodi H., "Solving a multi-order fractional differential equation using homotopy analysis method", Journal of King Saud University-Science, 23 (2) (2011) 151155.

26. Ganjiani M., "Solution of nonlinear fractional differential equations using homotopy analysis method", Applied Mathematical Modelling, 34(6) (2010) 1634-1641.

27. Saha Ray S., Patra A., "Haar wavelet operational methods for the numerical solutions of fractional order nonlinear oscillatory Van der Pol system", Applied Mathematics and Computation, 220 (2013) 659-667.

28. Konuralp A., Konuralp C., Yildirim A., "Numerical solution to the van der Pol equation with fractional damping", Physica Scripta, doi:10.1088/0031-8949/2009/T136/014034.

29. Saha Ray S., "On Haar wavelet operational matrix of general order and its application for 
the numerical solution of fractional Bagley Torvik equation", Applied Mathematics and Computation, 218 (2012) 5239-5248.

30. Mashayekhi S., Ordokhani Y., Razzaghi M., "Hybrid functions approach for nonlinear constrained optimal control problems", Communications in Nonlinear Science and Numerical Simulation, 17 (2012) 1831-1843.

31. Mashayekhi S., Ordokhani Y., Razzaghi M., "Hybrid functions approach for optimal control of systems described by integro-differential equations", Applied Mathematical Modelling, 37 (2013) 3355-3368.

32. Mashayekhi S., Razzaghi M., Tripak O., "Solution of the Nonlinear Mixed VolterraFredholm Integral Equations by Hybrid of Block-Pulse Functions and Bernoulli Polynomials", The Scientific World Journal, doi:10.1155/2014/413623.

33. Podlubny I., "Geometric and physical interpretation of fractional integration and fractional differentiation", Fractional Calculus and Applied Analysis, 5 (2002) 367-386.

34. Podlubny I., "Fractional Differential Equations: An Introduction to Fractional Derivatives", Fractional Differential Equations, to Methods of Their Solution and Some of Their Applications. New York: Academic Press (1998).

35. Kilbas A. A., Srivastava H. M, Trujillo J. J., "Theory and Applications of Fractional Differential Equations", vol. 204. Elsevier: North-Holland Mathematics Studies (2006).

36. Keshavarz E., Ordokhani Y., Razzaghi M., "A numerical solution for fractional optimal control problems via Bernoulli polynomials", Journal of Vibration and Control, 22 (18) (2016) 3889-3903.

37. Gu J. S., Jiang W. S., "The Haar wavelets operational matrix of integration", International Journal of Systems Science, 27 (1996) 623-628.

38. Keshavarz E., Ordokhani Y., Razzaghi M., "Bernoulli wavelet operational matrix of fractional order integration and its applications in solving the fractional order differential equations", Applied Mathematical Modelling, 38 (24) (2014) 6038-6051.

39. Costabile F., Dellaccio F., Gualtieri M. I., "A new approach to Bernoulli polynomials", Rendiconti di Matematica, Serie VII, 26 (2006) 1-12.

40. Arfken G., "Mathematical Methods for Physicists, Third edition", San Diego: Academic press (1985).

41. Kreyszig E., "Introductory Functional Analysis with Applications", New York: John Wiley 
and Sons Press, 1978.

42. Kilicman A., Al Zhour Z. A. A., "Kronecker operational matrices for fractional calculus and some applications", Applied Mathematics and Computation, 187 (1) (2007) 250-265.

43. Chen J-H., Chen W-C., "Chaotic dynamics of the fractionally damped van der Pol equation", Chaos Solitons Fractals, 35 (2008) 188-198.

44. Arikoglu A., Ozkol I., "Solution of fractional differential equations by using differential transform method", Chaos Solitons and Fractals, 34 (2007) 1473-1481.

45. O’Nan M., "Linear Algebra, Third edition", Harcourt College (1990).

\section{Appendix}

Lemma 1. If $T$ is a matrix with square submatrices as

$$
T=\left[\begin{array}{ll}
T_{1} & Z_{2} \\
Z_{1} & T_{2}
\end{array}\right]
$$

where the $Z$ 's are blocks of zeroes, then [45]

$$
|T|=\left|T_{1}\right| \cdot\left|T_{2}\right| \cdot
$$

In section 2 and $3, D$ and $\Phi$ are diagonal block matrices. If blockes on the main diagonal are vertible matrices, then using Lemma 1 , there are $D^{-1}$ and $\Phi^{-1}$. 